\title{
Self-similar and charged radiating spheres: an anisotropic approach
}

\author{
W. Barreto - B. Rodríguez • L. Rosales • \\ O. Serrano
}

Published online: 8 February 2007

(C) Springer Science+Business Media, LLC 2007

\section{Erratum to: Gen Relativ Gravit 39:23-29 DOI 10.1007/s10714-006-0365-3}

Unfortunately, several errors occurred in the published version of this article.

- The affiliation of the corresponding author W. Barreto was incorrect. The correct information is given below.

- In the $3^{\text {rd }}$ paragraph on p. 25 a wrong citation [35-41] was given. The complete paragraph with the correct citation is printed below:

Few exact solutions to the Einstein-Maxwell equations are relevant to gravitational collapse. For this reason, new collapse solutions are very useful, even if they are simplified ones [31]. It is well known that the field equations admit

The online version of the original article can be found at http://dx.doi.org/10.1007/s10714-006-0365-3.

W. Barreto $(\varangle)$

Departamento de Física, Centro de Física Fundamental, Facultad de Ciencias, Universidad de Los Andes, Mérida, Venezuela

e-mail: wbarreto@ula.ve

B. Rodríguez

Computational Science Research Center, College of Sciences, San Diego State University,

San Diego, CA, USA

L. Rosales

Laboratorio de Física Computacional, Universidad Experimental Politécnica

"Antonio José de Sucre", Puerto Ordaz, Venezuela

O. Serrano

Departamento de Ciencia y Tecnología, Universidad Experimental de Guayana,

Puerto Ordaz, Venezuela 
homothetic motion [32-34]. Applications of self-similarity range from modeling black holes to producing counterexamples to the cosmic censorship conjecture [35-46]. It is well established that in the critical gravitational collapse of an scalar field the space-time can be self-similar [47-49]. We can expect on physical grounds that this scenario remains similar for charged matter [50].

- In the reference list there were errors in reference nos. 1, 20, 21 and 60. The corrected references are printed below:

\section{References}

1. Ray, S., Espindola, A.L., Malheiro, M., Lemos, J.P.S., Zanchin, V.T.: Phys. Rev. D 68, 084004 (2003)

20. Barreto, W., Rojas, S.: Astrophys. Space Science 193, 201 (1992)

21. Barreto, W.: Astrophys. Space Science 201, 191 (1993)

60. Herrera, L., Ponde de León, J.: J. Math. Phys. 26, 2302 (1985) 